\title{
Habilidades Sociais e Desempenho Escolar em Português e Matemática em Estudantes do Ensino Fundamental
}

\author{
Daniel Bartholomeu \\ Núcleo de Pesquisa em Saúde e Desempenho Humano do Centro Universitário Fundação \\ Instituto para o Ensino de Osasco (FIEO), Osasco, SP, Brasil \\ José Maria Montiel ${ }^{1}$ \\ Soraia Néia \\ Marjorie C. Rocha Silva \\ Programa de Pós-Graduação em Psicologia Educacional do Centro Universitário FIEO, \\ Osasco, SP, Brasil
}

\begin{abstract}
Resumo
No presente trabalho verificou-se a relação das habilidades sociais e desempenho escolar em diferentes anos escolares e por sexo, visando fornecer evidências de validade para o Teste de Habilidades Sociais em Crianças e Adolescentes em situação escolar (THAS-C). Foram avaliadas 196 crianças do Ensino Fundamental de duas escolas do interior do estado de São Paulo, com idades que variaram de 8 a 10 anos, sendo 52\% do sexo feminino. Foi aplicado o Teste de Habilidades Sociais para crianças em situação escolar e o Teste de Desempenho de Escrita, Leitura e Matemática. Dentre os resultados, se verificou que um repertório maior de habilidades sociais tende a ser acessado em anos escolares mais avançados e parecem facilitar o desempenho em diferentes áreas de desempenho igualmente. Os resultados apresentam consonância com a literatura, indicando a necessidade de se ampliar as informações acerca do repertório social em crianças com diferentes desempenhos escolares. Neste sentido, pode-se aferir que as condutas se associam às dificuldades específicas em escrita, leitura e matemática e, que o curso destas associações varia em razão do sexo e idade. Além disso, se verificou a importância da avaliação e consideração de estudos sobre as habilidades sociais, dadas as implicações educacionais associadas.
\end{abstract}

Palavras-chave: Avaliação escolar, desempenho escolar, déficits em habilidades sociais.

\section{Social Skills and School Performance in Portuguese and Mathematics in Elementary School Students}

\begin{abstract}
In this study, the relations between social skills and academic performance were studied. We posed associations between social skills and performance in writing and mathematics as well as between conversational skills and these two academic performance measures in girls. In boys we posed associations between assertiveness and academic performance in the two areas. Participants were 196 elementary school children from two schools in the state of São Paulo, with ages ranging 8-10 years, 52\% females were evaluated by means of the Test of Social Skills in Children and Adolescents in school situation (THAS-C) and Test of academic performance in writing and maths. The results pointed out that greater

Endereço para correspondência: R. Leonita Faber Ladeira, 762, Jd. Estádio, Jundiaí, SP, Brasil 13202-010. E-mail: d_bartholomeu@yahoo.com.br, montieljm@hotmail.com, projeto_neuropsicologia@yahoo.com.br e silvamarjorie@yahoo.com.br
\end{abstract}


social skills tend to be achieved in later schooling years and appear to facilitate performance in different areas of academic performance. In this sense, we suggest that the behaviors are associated with specific difficulties in writing and mathematics, and that the course of these associations varies within sex and age. Furthermore, is indicated the importance of the evaluation of social skills in the academic context given the associated educational connections.

Keywords: Academic assessment, school performance, social skills.

\section{Habilidades Sociales y Rendimiento Escolar en Portugués y Matemáticas en los Estudiantes de Enseñanza Primaria}

\section{Resumen}

En el presente trabajo fue examinada la relación entre las habilidades sociales y el rendimiento académico en diferentes años escolares y género. Se utilizó la prueba habilidades sociales para niños en situación escolar (THAS-C). Este estudio evaluó 196 niños de la escuela primaria de dos escuelas en el estado de São Paulo. La edad Los participantes van de 8-10 años, 52\% mujeres. Para evaluar a los participantes se utilizó La prueba habilidades sociales para niños en situación escolar y la Prueba de Escritura de rendimiento, Lectura y Matemáticas. Entre los resultados, se encontró que el aumento de las habilidades sociales tiende a ocurrir más en años avanzados y para facilitar la operación igualmente en diferentes áreas. Los resultados muestran un acuerdo con la literatura, lo que indica la necesidad de ampliar la información acerca de las habilidades sociales de los niños en los niños con diferentes problemas de aprendizaje. En tal sentido, podríamos inferir que las conductas se asocian a dificultades específicas en la lectura, escritura y matemáticas y que el curso de estas asociaciones varía en función del sexo y la edad. Además, es importante que las habilidades sociales sean estudiadas en esta población, dada las implicadas educaciones asociadas.

Palabras clave: Evaluación de escolar, el rendimiento académico, déficits de las habilidades sociales.

De acordo com os apontamentos de Almeida et al. (1995) o fracasso escolar é um fenômeno recorrente na história educacional brasileira, constituindo-se um crônico e permanente desafio para psicólogos, pedagogos e profissionais de áreas afins já que decorre de uma constelação de fatores de ordem pessoal, familiar, emocional, pedagógica e social. Dentre estes, as interações sociais são entendidas como educativas na medida em que representam condições para a aquisição de conceitos e estratégias cognitivas que afetam o desenvolvimento social e escolar (Coll \& Sole, 1995; Del Prette \& Del Prette, 1997). As interações sociais satisfatórias da criança com colegas e professores requerem um repertório adequado de habilidades sociais de todas as partes envolvidas, ou seja, de diferentes classes de comportamentos sociais para lidar de maneira adequada com as demandas das situações interpessoais (Del Prette \& Del Prette,
2001, 2003; Del Prette \& Del Prette, 2005). São cada vez maiores os esforços em investigar os fatores subjacentes ou relacionados às dificuldades acadêmicas em leitura, escrita e matemática. E nessa perspectiva, as dificuldades em habilidades sociais são definidas como déficits de aquisição ou de desempenho adequado às demandas de interação da situação e da cultura e interferem, portanto, na qualidade dessas relações (Del Prette \& Del Prette, 2005; Gresham, 2009; Malecki \& Elliott, 2002; McClelland, Morrison, \& Holmes, 2000).

Dentre os fatores psicossociais relacionados ao desempenho escolar estão os conceitos de autoeficácia, motivação para aprender, crenças (Medeiros, Loureiro, Linhares, \& Marturano, 2000, 2003), aspectos afetivos (Loureiro, Marturano, Linhares, Machado, \& Silva, 1994), fatores da escola, ambiente de aprendizagem, tipo e intensidade da instrução, características 
do professor e recursos no ambiente familiar, como capacidade de lidar com os desafios da integração à escola, envolvimento parental com a escolarização do filho, presença de recursos no ambiente físico, práticas educativas e disciplina (Marturano, 1999). Ainda, variáveis sócio-econômicas (Leybaert, Alégria, Deltour, \& Skinkel, 1997; Yavas \& Haase, 1988), ambiente sociocultural (Witter, 1996) e variáveis sociolinguísticas (Tasca, 2002), como nível de escolaridade, classe socioeconômica e instrução específica sobre regras de escrita, estão relacionadas ao desempenho das crianças em leitura e escrita.

Segundo Gardinal e Marturano (2007), a qualidade avaliada (positiva ou negativa) das relações sociais estabelecidas entre as crianças em sala de aula na escola de Educação Infantil pode afetar o aprendizado das mesmas, sendo que as relações positivas em sala de aula estão associadas ao bom desempenho em leitura e escrita. Pesquisas nesta área (Bartholomeu et al., 2009; Del Prette \& Del Prette, 2003; Gresham, 2009) têm mostrado que as crianças com baixo desempenho escolar apresentam características interpessoais que incluem, entre outros aspectos, tendência a serem mais agressivas, a apresentarem interações mais negativas com companheiros, a terem mais problemas de personalidade, menos comportamentos orientados para tarefa e a apresentarem um repertório menos elaborado de comportamentos interpessoais apropriados e desejáveis socialmente, em comparação com crianças sem essas dificuldades (Marturano, Linhares, \& Parreira, 1993; Pinheiro, Haase, Del Prette, Amarante, \& Del Prette, 2006). Na avaliação dos professores (Maluf \& Bardelli, 1991), os alunos com dificuldades escolares são referidos como inquietos, briguentos, inibidos e sem iniciativa, com déficits nas habilidades de desenvolver e manter amizades, encerrar conversação, compartilhar brincadeiras e interagir com colegas.

Diferentes estudos enfocando a avaliação pelos colegas também demonstraram que os alunos com menor desempenho escolar são menos aceitos que seus colegas sem essas dificuldades (Bartholomeu, Montiel, \& Pessotto, 2011; Sto- ne \& La Greca, 1990; Vaughn \& Hogan, 1990; Vaughn, McIntosh, \& Spencer-Rowe, 1991). Segundo os apontamentos de Gresham, Lane, MacMillan, Bocian e Ward (2000) e Kavale e Forness (1996) citam os companheiros de sala como fonte importante de informações sobre o repertório social de crianças com problemas de comportamento e dificuldades escolares. Molina e Del Prette (2006) e Renk e Phares (2004) consideram os pais como informantes qualificados das características socioemocionais dessas crianças. Porém, existem autores que citam contextos em que crianças com dificuldades escolares podem não apresentar deficiências nas habilidades sociais (Elliott, Pring, \& Bunning, 2002; Gresham, 2009; Swanson \& Malone, 1992). Deste modo, Gresham (1992) sugere três hipóteses que podem nortear os estudos entre habilidades sociais e dificuldades escolares: a hipótese causal, na qual os déficits em habilidades sociais e a incapacidade de aprendizado seriam atribuídos à disfunção do sistema nervoso central; a hipótese da concomitância entre deficiência em habilidades sociais e dificuldades de aprendizado; e a hipótese correlacional, ou seja, uma tendência de parte dos alunos com maiores escores em habilidades sociais apresentarem melhor rendimento escolar.

Segundo estudos (Kavale \& Forness, 1996; Medeiros \& Loureiro, 2004; Swanson \& Malone, 1992) uma detalhada avaliação e monitoramento das dificuldades interpessoais e escolares, precocemente apresentadas pelas crianças, pode ser importante para o planejamento de estratégias pedagógicas e efetivas. Alguns autores tendem a considerar os déficits no repertório de habilidades sociais como fator de risco para a realização acadêmica e para o desenvolvimento socioemocional (Del Prette \& Del Prette, 2005; Gresham, 2009) que podem se agravar na adolescência e idade adulta (Caprara, Barbaranelli, Pastorelli, Bandura, \& Zimbardo, 2000; DiPerna \& Elliott, 2000; Malecki \& Elliott, 2002). A avaliação de habilidades sociais de crianças deve, na medida do possível, ser realizada por meio de um delineamento multimodal, ou seja, com base em diferentes procedimentos (entrevistas, inventários, observação) instrumentos e informantes 
(pais, professores, alunos e companheiros de sala; Del Prette \& Del Prette, 2009; Del Prette \& Del Prette, 2005; Gresham \& Elliott, 2008).

$\mathrm{Na}$ literatura nacional e internacional, de forma geral, as habilidades sociais são apontadas como importantes indicadores do desempenho escolar, e assim, altamente correlacionadas de forma positiva (por exemplo, Feitosa, Del Prette, \& Del Prette, 2012 e Feitosa, Del Prette, Del Prette, \& Loureiro, 2011). Alguns autores discutem que é possível que as habilidades sociais tenham elevada relação com o desempenho escolar em anos críticos de transição do ensino médio para a faculdade (Comedis, 2014). No entanto, a direção e os processos implicados nesta associação entre habilidades sociais e desempenho escolar não estão suficientemente claros quando se pensa nos vários níveis escolares e por sexo, revelando a necessidade de mais estudos na área sobretudo considerando estas variáveis (Bloom, Karagiannakis, Toste, Heath, \& Konstantinopoulos, 2007). Também a revisão de literatura extensa e sistemática de Gifford-Smith e Brownell (2003) indicou que a maior parte dos estudos que envolvem as habilidades sociais e a aceitação e rejeição entre colegas concentram-se em crianças e com foco maior na rejeição, com uma quantidade menor de estudos sobre a aceitação. Enfatizam ainda que a maior parte dos estudos sobre a sociometria concentram-se até a década de 90, com uma concentração menor de estudos a partir desta data.

Cabe realizar alguns destaques de estudos com crianças do Ensino Fundamental. Del Prette e Del Prette (2003) indicaram que de $20 \%$ a $42 \%$ das crianças com dificuldades de leitura, escrita e matemática apresentaram indicativos dos seguintes problemas de comportamento: distrai-se facilmente; briga com os outros; discute com os outros; mostra-se irrequieto ou se mexe excessivamente; baixa autoestima; interrompe a conversa dos outros; perturba as atividades em andamento; fica com raiva facilmente; ameaça ou intimida os outros; retruca quando os adultos lhe corrigem e age impulsivamente. Como pode ser observado, tais apontamentos se devem mais a problemas de comportamento do tipo externalizante (Del Prette \& Del Prette, 2003; Kavale
\& Forness, 1996). A alta frequência de comportamentos internalizantes pode comprometer as relações de amizade e importantes comportamentos orientados para a aprendizagem escolar como, por exemplo, fazer perguntas, tirar dúvidas e pedir ajuda de colegas e professores. A alta frequência de comportamentos externalizantes também compromete a qualidade das relações das crianças com seus professores e pares, bem como a adaptação à escola. Esses resultados vão ao encontro de pesquisas anteriores (Gresham, Lane, McMillan, \& Bocian, 1999; Gresham et al., 2000) que apontam baixo ajustamento escolar em crianças com comorbidade de rebaixamento escolar e problemas de comportamento.

Segundo dados obtidos por Bandeira Rocha, Pires, Del Prette e Del Prette (2006) em educandos do Ensino Fundamental I, crianças de escolas particulares e nível socioeconômico mais elevado, possuem níveis de competência acadêmica mais elevada. Além deste fator, pode-se também citar que as habilidades sociais da criança estão diretamente ligadas ao seu nível de competências e reprovações. O estudo de Montiel, Bartholomeu, Lima, Guidetti, e Machado (2011) relacionou dificuldades na escrita e habilidades sociais em crianças e revelou que somente o fator civilidade e altruísmo associou-se negativamente às dificuldades de escrita, sugerindo que quanto mais altruístas as crianças são, menos tendem a cometer erros de escrita.

Como se pode notar, existem na literatura indicações das relações entre o desempenho escolar e as habilidades sociais. Com isso, novos instrumentos que avaliem as habilidades sociais também devem demonstrar suas associações com o desempenho escolar, sendo estas uma evidência de validade; já que, como afirma Messick (2000), a validade de um instrumento é informada a partir da representação e das relações dos construtos teóricos nos instrumentos. Neste sentido, este estudo tem como objetivo estabelecer as relações entre as habilidades sociais em crianças e desempenho na leitura, escrita e matemática, visando fornecer evidências de validade para o Teste de Habilidades Sociais em Crianças e Adolescentes do Ensino Fundamental (THAS-C). Supõe-se que os escores da 
medida padronizada de desempenho em leitura, escrita e matemática irão associar-se com os resultados do THAS-C nas diferentes séries e sexo estudados, já que é relevante demonstrar as associações destes construtos nestes diferentes subgrupos (Anastasi \& Urbina, 2000). Além disso, as habilidades sociais tendem a associarem-se diferentemente nos indicadores de coesão grupal em diferentes anos escolares e sexo. Ora, a coesão grupal tende a ser associada também ao bom desempenho escolar, aventando-se a hipótese de que a associação entre habilidades sociais e o desempenho na escrita, leitura e matemática também seria, portanto, diferente em razão destes subgrupos (Bloom et al., 2007; Comedis, 2014; Gifford-Smith \& Brownell, 2003).

Espera-se que a civilidade e altruísmo estejam associados positivamente ao desempenho na escrita, leitura e matemática, sobretudo na escrita, em que outros estudos da literatura já evidenciaram estas relações (Montiel et al., 2011). Também se supõe que as habilidades de conversação e desenvoltura social estarão mais associadas positivamente ao desempenho escolar nas três áreas, considerando que déficits nas habilidades de desenvolver e manter amizades, encerrar conversação, compartilhar brincadeiras e interagir com colegas são características de crianças com dificuldades de aprendizagem, além do fato de que estes déficits tendem a comprometer as relações de amizade e importantes comportamentos orientados para a aprendizagem escolar como, por exemplo, fazer perguntas, tirar dúvidas e pedir ajuda de colegas e professores (Del Prette $\&$ Del Prette, 2003; Del Prette \& Del Prette, 2005; Gresham, 2009; Gresham et al., 1999; Gresham et al., 2000; Pinheiro et al., 2006).

\section{Método}

\section{Participantes}

Participaram 196 crianças de duas escolas de Ensino Fundamental do interior do estado de São Paulo, sendo uma particular $(N=72)$ e outra estadual $(N=124)$. O nível socioeconômico médio das escolas foi 2 salários (aproximadamente $\mathrm{R} \$ 1600,00$ mensais). A coleta ocorreu no final de 2010 e as crianças tinham idade que variavam de 8 anos e 4 meses a 10 anos e sete meses, com média de 9 anos e cinco meses e desvio padrão de 0,834 , sendo que $52 \%$ das crianças eram do sexo feminino, provenientes do $4^{\circ}(N=88)$ e $5^{\circ}$ ano escolar $(N=83)$.

\section{Instrumentos}

Teste de Habilidades Sociais em Crianças para o Ensino Fundamental (THAS-C; Bartholomeu, Silva, \& Montiel, 2014). Este instrumento foi desenvolvido a fim de mensurar habilidades sociais especificamente voltadas ao contexto escolar em crianças. Foram apresentados ao examinando 23 itens para serem respondidos quanto à frequência com que a criança se comporta da forma descrita nos itens com respostas numa escala Likert de três pontos. As categorias de resposta foram Nunca, Às vezes e Sempre e atribuiu-se pontos de 1 a 3 respectivamente a cada uma delas na correção do instrumento que foram somados. Alguns itens são pontuados de forma inversa, já que a conduta socialmente hábil está definida no oposto do que a frase propõe. Ao se hierarquizar a dificuldade dos itens, identificando quais condutas são mais fáceis de se apresentar no contexto de interação social, possibilita-se planejar intervenções para pessoas que apresentam baixa habilidade social, apresentando inicialmente condutas mais fáceis de a pessoa emitir, para então passar a comportamentos mais complexos, permitindo uma avaliação precisa do repertório comportamental da criança, possibilitando um escalonamento comportamental.

Os resultados da análise preliminar feita por componentes principais e rotação Varimax (com 257 sujeitos) sugeriram a estrutura de três fatores mais evidentes que explicaram $34,17 \%$ de variância. $\mathrm{O}$ primeiro fator foi denominado Civilidade e Altruísmo e apresentou um índice alfa de Cronbach de 0,87; o segundo, Desenvoltura e autocontrole na interação social obteve um coeficiente de 0,84; e o terceiro Assertividade com enfrentamento evidenciou um alfa de 0,60 . Esta estrutura foi replicada em um estudo posterior (relatado no manual citado) com 1381 sujeitos.

A primeira dimensão, Civilidade e altruísmo, abrange habilidades como agradecer elo- 
gios, pedir desculpas, ajudar os amigos, elogiá-los, expressar sentimentos positivos aos pares, ser educado ao manifestar uma opinião. No segundo fator, Desenvoltura e autocontrole na interação social, seus indicadores devem ser invertidos para indicarem tais aspectos. As situações propostas sugerem contextos negativos em que a criança é exposta a condutas sociais novas, desconhecidas ou que podem provocar constrangimento como receber críticas, falar para a sala toda, encerrar uma conversa, apresentar-se a um grupo de desconhecidos ou perguntar-lhes algo. Finalmente, a terceira dimensão, recebeu a denominação de Assertividade com enfrentamento. As habilidades propostas incluem demonstrar desagrado, defender seus direitos e opiniões, resistir à pressão do grupo, afirmando sua autoestima, sob o risco de uma reação indesejável por parte do interlocutor. A análise de Rasch, aplicada a esses itens, identificou o nível de dificuldade dos mesmos, possibilitando observar que os indicadores avaliam habilidades sociais medianas, embora o nível de dificuldade dos itens aumente de um fator para o outro. Também o ajuste dos itens restantes da escala por Rasch revelou que nenhum deles esteve fora dos limites aceitáveis quanto ao modelo de Rasch.

Teste de Desempenho de Escrita, Leitura e Matemática para o Ensino Fundamental (Silva, Montiel, Pires, \& Bartholomeu, 2010). Também foi aplicado o Teste de Desempenho de Escrita, Leitura e Matemática para o Ensino Fundamental. O teste foi desenvolvido a fim de avaliar a capacidade de leitura e interpretação de pequenos textos; escrita de palavras ditadas e a partir de figuras e execução de operações aritméticas dadas em formas de expressão e por meio de resolução de problemas, e assim indicar possíveis lacunas na aprendizagem; e quais áreas poderiam necessitar de maior acompanhamento escolar. O teste foi criado inicialmente para avaliação do $2^{\circ}$ ao $9^{\circ}$ ano do Ensino Fundamental visto que abarca itens com habilidades básicas e mais complexas, sendo divididas em fáceis, médias e difíceis. Este instrumento apresentou índices de precisão por Kuder Richardson (amostra de 251 crianças) variando entre 0,85 e 0,92 para seus fatores (ditado, nomeação, problemas, feitos com cálculos propostos de forma escrita, e contas, em que eram propostas somente as equações para solução) que demonstraram unidimensionalidade pelo modelo de Rasch (cada um deles separadamente). A análise fatorial pelo programa Testfact indicou a existência destes quatro fatores que explicaram juntos $45 \%$ de variância e a análise bi fatorial evidenciou dois fatores um associado à matemática e outro à leitura e escrita. Vale ressaltar que estes resultados estão em um relatório técnico deste instrumento que consta com suas análises psicométricas preliminares e seu manual está em confecção.

\section{Procedimento}

A aplicação dos instrumentos ocorreu de forma coletiva e foi realizada nas salas de aulas dos alunos cujos pais haviam autorizado previamente por meio da assinatura ao Termo de Consentimento Livre e Esclarecido (sob o número no comitê de ética 424-2010). Não houve seleção prévia dos alunos e participaram todos aqueles que os pais autorizaram, sendo uma amostragem por conveniência. Não participaram da pesquisa crianças de inclusão, já que se desejava obter uma amostra de crianças sem diagnóstico pré-estabelecido. Foi inicialmente explicado às crianças de que se tratava de pesquisa e como responder a cada um dos instrumentos. As questões foram lidas uma a uma pelo pesquisador que aguardava a resposta antes de prosseguir a coleta, sendo aplicado primeiramente o THAS-C. As crianças que não desejavam prosseguir tinham essa liberdade, sendo liberadas da aplicação.

\section{Resultados e Discussão}

Os coeficientes de correlação entre o desempenho escolar em leitura, escrita e matemática avaliados pelo teste de desempenho e habilidades sociais avaliadas pelo THAS-C no geral e separadamente por subgrupos (idade, sexo e ano escolar, respectivamente) estão nas Tabelas $1,2,3$ e 4 . 
Por esses dados, identificou-se que o desempenho na escrita por ditado e por figuras está associado moderado e positivamente com o altruísmo e de forma fraca e positiva com a habilidade social em geral. Isso facilita a interpretação de que, com o aumento das habilidades relativas à Civilidade e Altruísmo, tem-se uma tendência de aumento correspondente no desempenho em escrita, tanto por ditado quanto por figuras. Este achado já havia sido identificado por Montiel et al. (2011) em que os autores evidenciaram esta mesma associação empregando outro instrumento de avaliação de problemas de aprendizagem na escrita (ADAPE). Neste sentido, aparentemente as habilidades relacionadas ao altruísmo e civilidade estão relacionadas a um melhor aprendizado de escrita.

Especificamente, o fator assertividade se mostrou associado positivamente, porém de forma fraca com o desempenho em escrita por figuras e de forma moderada na resolução de problemas aritméticos, o que demonstra uma tendência de que as crianças mais assertivas tenham mais facilidade em se desempenhar principalmente na faceta da aritmética. Tal aspecto confirma os resultados de (Del Prette \& Del Prette, 2003;
Gresham et al., 1999; Gresham et al., 2000) em que apontam uma maior incidência de condutas externalizantes como pedir ajudas e tirar dúvidas, associadas à assertividade e ao bom desempenho escolar. É interessante observar que nas questões aritméticas que demandam mais tempo, estratégias e funções como memória, a assertividade e capacidade de pedir ajuda aos colegas podem ser entendidas como condutas que facilitam o desempenho neste tipo de tarefa. Assim, crianças que tendem a se expressar e relatar dúvidas e pedir ajuda mais facilmente tendem a solucionar melhor estas tarefas. Tal fato abre espaço para novas pesquisas.

Também foi testada a relação entre habilidades sociais e desempenho, separadamente por ano escolar. A Tabela 2 apresenta os coeficientes de correlação de Pearson entre facetas de desempenho e do THAS-C separados por ano escolar. Essa análise foi pensada ao se considerar que as relações entre habilidades sociais e coesão grupal são distintas em diferentes fases do processo de escolarização, sendo esperadas diferenças também nas associações com o desempenho escolar em diferentes fases do período educacional (Gifford-Smith \& Brownell, 2003).

Tabela 1

Coeficiente de Correlação de Pearson entre Facetas de Desempenho e do THAS-C

\begin{tabular}{cccccc}
\hline Facetas do desempenho & Altruísmo & $\begin{array}{c}\text { Conversação e } \\
\text { desenvoltura social }\end{array}$ & Assertividade & $\begin{array}{c}\text { Habilidade social } \\
\text { geral }\end{array}$ \\
\hline Escrita por ditado & $r$ & $0,40^{*}$ & $-0,14$ & 0,19 & $0,31^{*}$ \\
& $p$ & 0,01 & 0,17 & 0,06 & 0,01 \\
Escrita por figuras & $r$ & $0,38^{*}$ & $-0,12$ & $0,22^{*}$ & $0,31^{*}$ \\
& $p$ & 0,01 & 0,28 & 0,04 & 0,01 \\
Ordem numérica & $r$ & 0,09 & $-0,09$ & $-0,01$ & 0,02 \\
Operações Aritméticas & $p$ & 0,39 & 0,38 & 0,94 & 0,83 \\
& $r$ & 0,34 & 0,07 & 0,11 & 0,36 \\
Problemas Aritméticos & $r$ & 0,07 & 0,71 & 0,57 & 0,06 \\
& $p$ & 0,17 & $-0,08$ & $0,44^{*}$ & 0,23 \\
\hline
\end{tabular}

Nota. * Correlação significativa. 
Tabela 2

Coeficientes de Correlação de Pearson entre Facetas de Desempenho e do THAS-C Separados por Ano Escolar

\begin{tabular}{|c|c|c|c|c|c|c|}
\hline Ano & Facetas do desempenho & & Altruísmo & $\begin{array}{c}\text { Conversação e } \\
\text { desenvoltura social }\end{array}$ & Assertividade & $\begin{array}{l}\text { Habilidade } \\
\text { Social geral }\end{array}$ \\
\hline \multirow[t]{10}{*}{4} & Escrita por ditado & $r$ & $0,42 *$ & $-0,10$ & 0,15 & $0,34 *$ \\
\hline & & $p$ & 0,01 & 0,51 & 0,34 & 0,02 \\
\hline & Escrita por figuras & $r$ & $0,45^{*}$ & $-0,04$ & 0,16 & $0,40^{*}$ \\
\hline & & $p$ & 0,01 & 0,82 & 0,30 & 0,01 \\
\hline & Ordem numérica & $r$ & 0,26 & $-0,11$ & $-0,03$ & 0,14 \\
\hline & & $p$ & 0,07 & 0,46 & 0,82 & 0,34 \\
\hline & Operações Aritméticas & $r$ & 0,42 & 0,09 & $-0,10$ & 0,41 \\
\hline & & $p$ & 0,14 & 0,77 & 0,75 & 0,15 \\
\hline & Problemas Aritméticos & $r$ & 0,15 & 0,31 & $0,61 *$ & $0,56^{*}$ \\
\hline & & $p$ & 0,62 & 0,30 & 0,03 & 0,05 \\
\hline \multirow[t]{10}{*}{5} & Escrita por ditado & $r$ & $0,41 *$ & $-0,19$ & $0,35^{*}$ & $0,35^{*}$ \\
\hline & & $p$ & 0,01 & 0,22 & 0,02 & 0,02 \\
\hline & Escrita por figuras & $r$ & $0,33 *$ & $-0,34 *$ & $0,41 *$ & 0,19 \\
\hline & & $p$ & 0,04 & 0,04 & 0,01 & 0,24 \\
\hline & Ordem numérica & $r$ & $-0,04$ & $-0,09$ & 0,20 & $-0,03$ \\
\hline & & $p$ & 0,79 & 0,56 & 0,19 & 0,87 \\
\hline & Operações Aritméticas & $r$ & $0,77 *$ & $-0,29$ & $0,68 *$ & $0,64^{*}$ \\
\hline & & $p$ & 0,01 & 0,38 & 0,02 & 0,03 \\
\hline & Problemas Aritméticos & $r$ & 0,40 & $-0,23$ & 0,46 & 0,36 \\
\hline & & $p$ & 0,11 & 0,35 & 0,06 & 0,15 \\
\hline
\end{tabular}

Nota. ${ }^{*}$ Correlação significativa.

Nota-se que o desempenho no $4^{\circ}$ ano, tanto em escrita por ditado quanto por figuras, mostrase positiva e significativamente associado ao altruísmo e às habilidades sociais em geral. Assim, as relações identificadas com a amostra toda foram mantidas nos estudantes de quarto ano, excetuando-se as associações entre escrita por figuras e assertividade que não se manteve neste ano. No $5^{\circ}$ ano, verificaram-se associações positivas e significativamente diferentes de zero entre o desempenho em escrita por ditado e resolução de operações aritméticas com as habilidades de altruísmo, assertividade e habilidades sociais em geral. É interessante observar que um repertório maior de habilidades sociais esteve associado ao bom desempenho acadêmico tanto em escrita como em matemática neste ano escolar. Assim, a assertividade associou-se ao bom desempenho em escrita por ditado e as habilidades de conversação à escrita por figuras. Ainda em relação à matemática, a civilidade e altruísmo também se associaram ao bom desempenho em solução de problemas.

Tal fato sugere que um repertório maior de habilidades sociais tende a ser empregado em idades mais avançadas, o que parece facilitar o desempenho em diferentes áreas acadêmicas igualmente. Por um lado, isso pode ser devido à 
maior variabilidade tanto de repertórios de habilidades sociais como de desempenho em escrita e matemática nestes anos escolares. Por outro, fortalece a evidência de que um maior repertório de habilidades sociais tende a facilitar o aprendizado em diferentes áreas como sugerem outras pesquisas neste âmbito (Bartholomeu et al., 2009; Bartholomeu, Montiel, et al., 2011; Del Prette \& Del Prette, 2003; Montiel et al., 2011; Stone \& La Greca, 1990; Vaughn \& Hogan, 1990; Vaughn et al., 1991).

Como sugere Gresham (1992) existem correlações positivas entre bom desempenho social e escolar, mas nem todas as crianças que apresentam um tipo de repertório tendem a apresentar uma dificuldade, valendo a pena analisar a funcionalidade desta relação entre estes dois construtos. Em outras palavras, estas deficiências podem ser decorrentes de outras dificuldades que a criança esteja vivenciando e são indicadores de problemas no desenvolvimento socioemocional da criança que tendem a ser agravados na adolescência e vida adulta se não cuidados. Assim, quando as correlações entre habilidades sociais e desempenho escolar são baixas, outros fatores podem afetar esta relação, como problemas socioemocionais e devem ser mais bem investigados em casos de dificuldades de aprendizagem. Neste sentido, a literatura aponta que as habilidades sociais tendem a ser

Tabela 3

Coeficientes de Correlação de Pearson entre Facetas de Desempenho e do THAS-C Separados por Sexo

\begin{tabular}{|c|c|c|c|c|c|c|}
\hline Sexo & Facetas do desempenho & & $\begin{array}{l}\text { Civilidade } \\
\text { e Altruísmo }\end{array}$ & $\begin{array}{c}\text { Conversação e } \\
\text { desenvoltura social }\end{array}$ & Assertividade & $\begin{array}{l}\text { Habilidade } \\
\text { social geral }\end{array}$ \\
\hline \multirow[t]{10}{*}{ Masc } & Escrita por ditado & $r$ & $0,48 *$ & $-0,04$ & $0,36^{*}$ & $0,49 *$ \\
\hline & & $p$ & 0,01 & 0,81 & 0,02 & 0,01 \\
\hline & Escrita por figuras & $r$ & $0,39 *$ & $-0,07$ & $0,39 *$ & $0,41 *$ \\
\hline & & $p$ & 0,01 & 0,66 & 0,01 & 0,01 \\
\hline & Ordem numérica & $r$ & 0,19 & $-0,28$ & 0,29 & 0,11 \\
\hline & & $p$ & 0,21 & 0,07 & 0,05 & 0,46 \\
\hline & Operações Aritméticas & $r$ & $0,60 *$ & 0,03 & $0,76^{*}$ & $0,64^{*}$ \\
\hline & & $p$ & 0,04 & 0,93 & 0,00 & 0,03 \\
\hline & Problemas Aritméticos & $r$ & 0,45 & 0,31 & 0,40 & $0,54^{*}$ \\
\hline & & $p$ & 0,08 & 0,24 & 0,13 & 0,03 \\
\hline \multirow[t]{10}{*}{ Fem } & Escrita por ditado & $r$ & 0,27 & $-0,21$ & 0,05 & 0,11 \\
\hline & & $p$ & 0,06 & 0,15 & 0,71 & 0,44 \\
\hline & Escrita por figuras & $r$ & $0,35^{*}$ & $-0,14$ & 0,07 & 0,22 \\
\hline & & $p$ & 0,02 & 0,37 & 0,66 & 0,15 \\
\hline & Ordem numérica & $r$ & 0,04 & $-0,01$ & $-0,25$ & $-0,06$ \\
\hline & & $p$ & 0,81 & 0,93 & 0,08 & 0,69 \\
\hline & Operações Aritméticas & $r$ & 0,26 & 0,05 & $-0,22$ & 0,17 \\
\hline & & $p$ & 0,34 & 0,85 & 0,41 & 0,53 \\
\hline & Problemas Aritméticos & $r$ & $-0,02$ & $-0,38$ & 0,45 & $-0,11$ \\
\hline & & $p$ & 0,95 & 0,13 & 0,07 & 0,67 \\
\hline
\end{tabular}

Nota. * Correlação significativa. 
fatores de proteção para a realização acadêmica e o bom desenvolvimento socioemocional, dependendo da forma como se usam tais comportamentos, ou seja, da competência social (Del Prette \& Del Prette, 2005; Gresham, 2009).

O presente estudo também evidenciou coeficientes baixos entre essas medidas, de forma que a sugestão de Del Prette e Del Prette (2005) e Gresham (2009) de que outros fatores socioemocionais possam afetar a funcionalidade desta relação entre habilidades sociais e desempenho escolar é uma explicação plausível e que convida a novas investigações. Outra possibilidade analisada foram diferenças no padrão de associações entre essas variáveis por sexo, já que diferentes condutas sociais tendem a associarem-se diferentemente à coesão grupal em diferentes grupos (Gifford-Smith \& Brownell, 2003). Assim, a seguir são analisadas possíveis associações entre esses dois construtos, separando-se por sexo dos participantes. Os resultados são apresentados na Tabela 3 onde são apresentados os coeficientes de correlação de Pearson entre facetas de desempenho e do THAS-C separados por sexo.

Verifica-se por esses resultados que as maiores associações positivas e significativas aparecem mais separadamente para os participantes do sexo masculino, de forma que quanto maior o desempenho na escrita por palavras, figuras e nas operações matemáticas, há também maior tendência de aumento nas habilidades de Altruísmo, Assertividade e habilidades sociais em geral. Das hipóteses inicialmente aventadas acerca das relações entre essas variáveis por sexo, algumas somente foram confirmadas. De fato, no sexo masculino, a assertividade esteve associada ao desempenho tanto na escrita como em matemática, mas a civilidade e altruísmo também. Já no sexo feminino, somente civilidade e altruísmo associou-se ao desempenho em escrita por figuras, o que não se havia aventado inicialmente.

Seguindo as análises, verificaram-se quais das habilidades sociais melhor explicavam o desempenho em cada um dos aspectos da leitura, escrita e matemática avaliados. Para isso, empregou-se a análise de regressão linear com método Backward de entrada das variáveis tendo como variáveis dependentes os fatores de desempenho escolar e independentes as habilidades sociais. O diagnóstico de colinearidade revelou não existirem problemas neste sentido (índices de Tolerância e Variance Inflation Factor [VIF] dentro de parâmetros esperados).

A Tabela 4 apresenta os coeficientes de regressão para os modelos que apresentaram significância estatística. Por esses dados evidencia-se que o escore geral de habilidades sociais previu significativamente o desempenho nas provas de ditado e nomeação, enquanto o fator assertividade explicou o desempenho em problemas de matemática. Os coeficientes foram todos positivos e de baixos a moderados, sugerindo que a contribuição destes fatores de habilidades sociais, apesar de não ser tão grande para cada tipo de tarefa (sugerindo explicarem pouca variância), foram significativos. E, suas tendências sugerem que o aumento das habilidades sociais lhe corresponde um aumento do desempenho do ditado (escrita) e nomeação (leitura), bem como o aumento da assertividade é acompanhado pelo aumento de acertos em problemas matemáticos. 

do Ensino Fundamental.

Tabela 4

Análise de Regressão entre Facetas de Desempenho e do THAS-C

\begin{tabular}{|c|c|c|c|c|c|c|}
\hline \multirow[t]{2}{*}{ Modelo } & & \multicolumn{2}{|c|}{ ANOVA } & $\begin{array}{l}\text { Coeficientes } \\
\text { padronizados }\end{array}$ & \multirow{2}{*}{$l$} & \multirow{2}{*}{$p$} \\
\hline & & $F$ & $p$ & Beta & & \\
\hline \multirow[t]{2}{*}{3 Nomeação } & (Constant) & 3,80 & 0,05 & & 2,39 & 0,02 \\
\hline & Habilidade social geral & & & 0,21 & 1,95 & 0,05 \\
\hline \multirow[t]{2}{*}{3 Problemas } & (Constant) & 4,70 & 0,04 & & $-0,21$ & 0,83 \\
\hline & Assertividade & & & 0,36 & 2,17 & 0,04 \\
\hline \multirow[t]{3}{*}{2 Ditado } & $($ Constant $)$ & 5,57 & 0,01 & & 1,73 & 0,09 \\
\hline & Conversação e desenvoltura social & & & $-0,17$ & $-1,72$ & 0,09 \\
\hline & Habilidade Social geral & & & 0,27 & 2,68 & 0,01 \\
\hline
\end{tabular}

É interessante observar que somente a medida de habilidade social geral manteve-se no modelo explicativo do desempenho no ditado e nomeação. Neste sentido, uma quantidade maior de repertório de condutas sociais das crianças é necessária para que o desempenho nestas duas áreas covarie. Já nos problemas matemáticos, o aumento da assertividade pode ser sugerido como uma variável que apresente algum efeito sobre sua melhora. Com isso, a intervenção em habilidades sociais visando à melhora do desempenho escolar de crianças poderia tomar rumos e focos distintos em razão do tipo de dificuldade específica que a criança exiba.

O estudo de Bartholomeu et al. (2014) que examinou a estrutura interna do THAS sugere que os níveis de dificuldade das condutas sociais neste teste seja empregada como referencial para o planejamento de treinamento em habilidades sociais. Com isso, a melhora do desempenho na escrita e leitura deveria ser pensada num treinamento iniciando pelos elementos mais fáceis (altruísmo e civilidade) até os mais difíceis (assertividade), ao passo que no caso da matemática, aparentemente as habilidades mais difíceis de serem treinadas (como sugerido pelos autores) devem ser o foco da intervenção. Tal aspecto convida a novas investigações sobre a intervenção nestes dois âmbitos. Há que se considerar que os meninos tendem a apresentar mais dificuldades escolares que as meninas e que essas diferenças nas associações de habilidades sociais e desempenho escolar já tem sido identificados também em outros estudos como de Bandeira et al. (2006) e em Montiel et al. (2011). De fato, um maior repertório de habilidades sociais parece favorecer o desempenho, sobretudo de meninos em nível fundamental I de ensino. Em meninas, alguns aspectos (como o altruísmo) tendem a estarem associados ao desempenho escolar

De fato, problemas emocionais e sociais tendem a afetar mais o desempenho escolar em meninos que em meninas como já demonstraram outros estudos, tornando este resultado algo esperado (Bartholomeu, Sisto, \& Rueda, 2006). Todavia, estes aspectos devem ser considerados ao se planejar as intervenções no que se refere aos aspectos socioemocionais em crianças com dificuldades de aprendizagem. De fato, o presente trabalho revelou que as habilidades sociais tendem a associarem-se mais ao desempenho escolar em meninos e em crianças (de ambos os sexos) de séries mais avançadas (quinta série - Ciclo II do Ensino Fundamental). Novas 
investigações poderiam tentar inserir e ampliar o repertório social de crianças mais jovens para verificar se estas habilidades ao serem aprendidas precocemente tendem a facilitar o desempenho escolar em anos iniciais, partindo do suposto de Gresham (1992) e Gresham et al. (1999) que as habilidades sociais seriam protetivas quanto ao bom desempenho escolar. Isso demandaria estudos com delineamento experimental e com maior controle também, além de outras formas de coletas de informação acerca do repertório social da criança como pais e professores (Gresham, 1992; Gresham et al., 2000; Kavale \& Forness, 1996).

\section{Considerações Finais}

De modo geral, estes resultados estão em consonância com as sugestões de Molina e Del Prette (2006) e Renk e Phares (2004) que indicaram a necessidade de se ampliar as informações acerca do repertório social de crianças em crianças com diferentes dificuldades escolares. Neste sentido, indicou-se condutas que se associam a desempenhos específicos em escrita, leitura e matemática e que o curso destas associações varia em razão do sexo e idade, o que também encontra eco em outras pesquisas como Bandeira et al. (2006) e mais recentemente em Montiel et al. (2011).

Apesar de em algumas correlações terem sido baixas, estes resultados poderiam ser refletidos em termos de coesão grupal, visto que as habilidades sociais consistem de algumas das características que asseguram uma melhor coesão grupal a partir, principalmente, da redução de rejeição social (Bartholomeu, Carvalho, Silva, Miguel, \& Machado, 2011; Bartholomeu, Montiel, et al., 2011; Gifford-Smith \& Brownell, 2003). Neste sentido, estudos tem revelado que a boa coesão grupal tende a facilitar o desempenho escolar (Gifford-Smith \& Brownell, 2003). Assim, valeria a pena investigar se as correlações entre habilidades sociais e desempenho escolar não são mediadas pela coesão grupal, ou, em outros termos, pela aceitação e rejeição no grupo. Tal fato convida a novas investigações no tema.

Dentre as limitações deste trabalho está a quantidade reduzida de crianças em algumas medidas, o que impactou diretamente no nível de significância das correlações encontradas. Assim, alguns coeficientes moderados não alcançaram significância estatística e deveriam ser considerados como representativos da amostra em questão, mas não úteis para generalizações, não apresentando representação nomotética. Neste sentido, estudos com amostras maiores seriam possibilidades interessantes para a continuidade deste trabalho. Além disso, apesar da abordagem correlacional ser uma possibilidade para investigar os construtos em questão (como sugere Gresham, 1992) estudos mais controlados são necessários visando estabelecer o impacto diferencial de cada tipo de habilidade social para cada problema escolar em específico. O presente estudo apresenta um breve sumário (baseado nas correlações das medidas) das possibilidades de relação entre habilidades sociais e desempenho escolar, e aponta a importância de que o uso dos comportamentos social habilidosos possam denotar competência social, para então repercutir em possíveis melhorias de desempenho escolar.

De forma sucinta, a civilidade e altruísmo parecem estar mais associados à escrita, enquanto a assertividade mais associada ao desempenho em matemática. Essas relações são aumentadas e estendidas em anos mais avançados e no sexo masculino, indicando pontos específicos de intervenção em habilidades sociais visando a melhora acadêmica. Estudos com este tipo de intervenção são igualmente necessários. De um modo geral, as associações que frequentemente se tem evidenciado na literatura entre desempenho escolar e habilidades sociais foram reafirmadas neste estudo com o THAS-C, sendo uma evidência de validade para este teste, já que relações esperadas deste construto avaliado por este teste se mantiveram nesta medida (convergência de avaliação). Outras evidências de validade poderiam também ser analisadas quanto 
às relações com outras variáveis como com o teste sociométrico e outras medidas de habilidades sociais como o Inventário Multimídia de Habilidades Sociais para Crianças (IMHSC) -Del Prette, o que também revela necessidade de novas pesquisas.

No que se refere especificamente à leitura, matemática e escrita, os estudos que relacionam problemas de conduta e interações sociais e o desempenho escolar nessas áreas indicam que o conhecimento cientifico, adquirido em ambiente formal de aprendizagem só será efetivo e produtivo se o mesmo for saudável e agradável ao educando. Existe, sem dúvidas, uma intensa relação entre o ambiente escolar e problemas de aprendizagem, sejam eles quais forem. Deste modo a atuação de profissionais treinados e capacitados ao auxílio pedagógico, psicopedagógico e psicológico devem estar presentes. Convém que sejam realizados novos estudos com acompanhamentos longitudinais a fim de acompanhar o efeito das variáveis nos diversos anos escolares. Bem como seria interessante propor uma pesquisa experimental em que fosse aplicado um treinamento de habilidades sociais em crianças com baixo desempenho escolar, e assim verificar possíveis melhorias nesses aspectos.

\section{Referências}

Almeida, S. F. C., Rabelo, L. de M., Cabral, V. S., Moura, E. R. de O., Barreto, M. de S. F., \& Barbosa, H. (1995). Concepções e práticas de psicólogos escolares acerca das dificuldades de aprendizagem. Psicologia: Teoria e Pesquisa, 11(2), 117-134.

Anastasi, A., \& Urbina, S. (2000). Testagem psicológica (7. ed.). Porto Alegre, RS: ArtMed.

Bandeira, M., Rocha, S. S., Pires, L. G., Del Prette, Z. A. P., \& Del Prette, A. (2006). Competência acadêmica de crianças no Ensino Fundamental: Características sociodemográficas e relação com habilidades sociais. Interação em Psicologia, 10(1), 53-62.

Bartholomeu, D., Bartholomeu, L. L., Machado, A. A., Cozza, H. F. P., Berberian, A. A., \& Montiel,
J. M. (2009). Dificuldades de aprendizagem, personalidade, emoções e características psicossociais de crianças: Revisando a literatura e sugerindo modelos. In J. M. Montiel \& F. C. Capovilla (Eds.), Atualização em transtornos de aprendizagem (pp. 355-382). São Paulo, SP: Artes Médicas.

Bartholomeu, D., Carvalho, L. F., Silva, M. C. R., Miguel, F. K., \& Machado, A. A. (2011). Aceitação e rejeição entre pares e habilidades sociais em universitários. Estudos de Psicologia (Natal), 16(2), 155-162.

Bartholomeu, D., Montiel, J. M., \& Pessotto, F. (2011). Sociometria e habilidades sociais em alunos do ensino médio. Estudos Interdisciplinares em Psicologia, 2(2), 211-228. doi:10.5433/2236-6407.2011v2n2p211

Bartholomeu, D., Silva, M. C. R., \& Montiel, J. M. (2014). Teste de Habilidades Sociais para Crianças e Adolescentes em Situação EscolarTHAS-C. São Paulo, SP: Memnon.

Bartholomeu, D., Sisto, F. F., \& Rueda, F. J. M. (2006). Dificuldades de aprendizagem na escrita e características emocionais de crianças. Psicologia em Estudo, 11(1), 19-22. doi:10.1590/ S1413-73722006000100016

Bloom, E. L., Karagiannakis, A., Toste, J. R., Heath, N. L., \& Konstantinopoulos, E. (2007). Severity of academic achievement and social skills deficits. Canadian Journal of Education, 30(3), 911-930.

Caprara, G. V., Barbaranelli, C., Pastorelli, C., Bandura, A., \& Zimbardo, P. G. (2000). Prosocial foundations of children's academic achievement. Psychological Science, 11, 302-306. doi:10.1111/1467-9280.00260

Coll, C., \& Sole, I. (1995). A interação professor/aluno no processo de ensino aprendizagem. In C. Coll, J. Palácios, \& A. Marchesi (Eds.), Desenvolvimento psicológico e educação: Necessidades educativas especiais e aprendizagem escolar. Porto Alegre, RS: ArtMed.

Comedis, E. J. (2014). The drole of social skills in the academic performance of De La Salle Araneta University freshmen students: Creating a culture. Paper presented at the De La Salle University Research Congress, Manila, Philippines. Retrieved from http:// www.dlsu.edu.ph/conferences/dlsu_research_ 
congress/2014/ pdf/proceedings/LCCS-I-002FT.pdf

Del Prette, A., \& Del Prette, Z. A. P. (1997). Habilidades sociais e construção de conhecimento em contexto escolar. In D. R. Zamignani (Ed.), Sobre comportamento e cognição: A aplicação da analise do comportamento e da terapia cognitivo-comportamental no hospital geral e nos transtornos psiquiátricos (pp. 234-250). Santo André, SP: Arbytes.

Del Prette, A., \& Del Prette, Z. A. P. (2001). Psicologia das relações interpessoais: Vivências para o trabalho em grupo. Petrópolis, RJ: Vozes.

Del Prette, A., \& Del Prette, Z. A. P. (2003). Aprendizagem socioemocional na infância e prevenção da violência: Questões conceituais e metodologia da intervenção. In A. Del Prette \& Z. A. P. Del Prette (Eds.), Habilidades sociais, desenvolvimento e aprendizagem (pp. 83-127). Campinas, SP: Alínea.

Del Prette, A., \& Del Prette, Z. A. P. (2009). Componentes não verbais e paralinguísticos das habilidades sociais. In A. Del Prette \& Z. A. P. Del Prette (Eds.), Psicologia das habilidades sociais: Diversidade teórica e suas implicações (pp. 147-186). Petrópolis, RJ: Vozes.

Del Prette, Z. A. P., \& Del Prette, A. (2005). Psicologia das habilidades sociais na infância: Terapia e educação. Petrópolis, RJ: Vozes.

DiPerna, J. C., \& Elliott, S. N. (2000). ACES: The academic competence evaluation scales (ACES $K-12)$. San Antonio, TX: The Psychological Corporation.

Elliott, C., Pring, T., \& Bunning, K. (2002). Social skills training for adolescents with intellectual disabilities: A cautionary note. Journal of Applied Research in Intellectual Disabilities, 15, 91-96. doi:10.1046/j.1360-2322.2001.00091.x

Feitosa, F. B., Del Prette, Z. A. P., \& Del Prette, A. (2012). Social skills and academic achievement: The mediating function of cognitive competence. Temas em Psicologia, 20(1), 61-70.

Feitosa, F. B., Del Prette, Z. A. P., Del Prette, A., \& Loureiro, S. R. (2011). Explorando relações entre o comportamento social e o desempenho acadêmico em crianças. Estudos e Pesquisas em Psicologia, 11, 442-455.
Gardinal, E. C., \& Marturano, E. M. (2007). Meninos e meninas na Educação Infantil: Associação entre comportamento e desempenho. Psicologia em Estudo, 12, 541-551. doi:10.1590/S141373722007000300011

Gifford-Smith, M. E., \& Brownell, C. A. (2003). Childhood peer relationships: Social acceptance, friendships, and peer networks. Journal of School Psychology, 41, 235-284. doi:10.1016/ S0022-4405(03)00048-7

Gresham, F. M. (1992). Social skills and learning disabilities: Causal, concomitant or correlational? School Psychological Review, 21(3), 348-360.

Gresham, F. M. (2009). Análise do comportamento aplicada às habilidades sociais. In Z. A. P. Del Prette \& A. Del Prette (Eds.), Psicologia das habilidades sociais: Diversidade teórica e suas implicações (pp. 17-66). Petrópolis, RJ: Vozes.

Gresham, F. M., \& Elliott, S. N. (2008). Social skills improvement system: Rating scales. Bloomington, MN: Pearson Assessments.

Gresham, F. M., Lane, L. K., McMillan, D. L., \& Bocian, K. M. (1999). Social and academic profiles of externalizing and internalizing groups: Risk factors for emotional and behavioral disorders. Behavioral Disorders, 24(3), 231-245.

Gresham, F. M., Lane, K. L., MacMillan, D. L., Bocian, K., \& Ward, S. (2000). Effects of positive and negative illusory biases: Comparisons across social and academic self-concept domains. Journal of School Psychology, 38, 151-175. doi:10.1016/S0022-4405(99)00042-4

Kavale, K. A., \& Forness, S. R. (1996). Social skills deficits and learning disabilities: A meta-analisys. Journal of Learning Disabilities, 29(3), 226-237. doi:10.1177/002221949602900301

Leybaert, J., Alégria, J., Deltour, J., \& Skinkel, R. (1997). Aprender a ler: O papel da linguagem, da consciência fonológica e da escola. In J. Grégoire \& B. Piérart (Eds.), Avaliação dos problemas de leitura: Os novos modelos teóricos e suas implicações diagnósticas (pp. 143-166). Porto Alegre, RS: Artes Médicas

Loureiro, S. R., Marturano, E. M., Linhares, M. B. M., Machado, V. L. S., \& Silva, R. A. (1994). Crianças com queixa de dificuldade escolar: 
Avaliação psicológica através de técnica gráfica. Arquivos Brasileiros de Psicologia Aplicada, 46, 161-182.

Malecki, C. K., \& Elliott, S. N. (2002). Children's social behaviors as predictors of academic achievement: A longitudinal analysis. School Psychology Quarterly, 17(1), 1-23. doi:10.1521/ scpq.17.1.1.19902

Maluf, M. R., \& Bardelli, C. (1991). As causas do fracasso escolar na perspectiva de professoras e alunos de uma escola de primeiro grau. Psicologia: Teoria e Pesquisa, 7(3), 263-271.

Marturano, E. M. (1999). Recursos no ambiente familiar e dificuldades de aprendizagem na escola. Psicologia: Teoria e Pesquisa, 15, 153162. doi:10.1590/S0102-37721999000200006

Marturano, E. M., Linhares, M. B. M., \& Parreira, V. L. C. (1993). Problemas emocionais e comportamentais associados a dificuldades na aprendizagem escolar. Medicina (Ribeirão Preto), 26(2), 161-175.

McClelland, M. M., Morrison, F. J., \& Holmes, D. L. (2000). Children at risk for early academic problems: The role of learning-related social skills. Early Childhood Research Quarterly, 15, 307-329.

Medeiros, C. P., \& Loureiro, S. R. (2004). Observação clínica do comportamento de crianças com queixa de dificuldade de aprendizagem. In E. M. Marturano, M. B. M. Linhares, \& S. R. Loureiro, Vulnerabilidade e proteção. Indicadores na trajetória do desenvolvimento escolar (pp. $107-$ 136). São Paulo, SP: Casa do Psicólogo

Medeiros, P. C., Loureiro, S. R., Linhares, M. B. M., \& Marturano, E. M. (2000). A auto-eficácia e os aspectos comportamentais de crianças com dificuldade de aprendizagem. Psicologia: Reflexão e Critica, 13, 327-336. doi:10.1590/ S0102-79722000000300002

Medeiros, P. C., Loureiro, S. R., Linhares, M. B. M., \& Marturano, E. M. (2003). O senso de auto-eficácia e o comportamento orientado para aprendizagem em crianças com queixa de dificuldade de aprendizagem. Estudos de Psicologia (Natal), 8, 93-105. doi:10.1590/ S1413-294X2003000100011

Messick, S. (2000). Consequences of test interpretation and use: The fusion of validity and values in psychological assessment. In R. D. Goffin \& E. Helmes (Eds.), Problems and solutions in human assessment: Honoring Douglas $N$. Jackson at seventy (pp. 3-20). Boston, MA: Kluwer Academic.

Molina, R. C. M., \& Del Prette, Z. A. P. (2006). Funcionalidade da relação entre habilidades sociais e dificuldades de aprendizagem. Psico-USF, 11(1) 53-63. doi:10.1590/S141382712006000100007

Montiel, J. M., Bartholomeu, D., Lima, F. F., Guidetti, A. A., \& Machado, A. A. (2011). Dificuldades de aprendizagem na escrita e habilidades sociais em crianças do ensino fundamental. Revista Portuguesa de Dificuldades de Aprendizagem, 1, 23-32.

Pinheiro, M. I. S., Haase, V. G., Del Prette, A., Amarante, C. L. D., \& Del Prette, Z. A. P. (2006). Treinamento de habilidades sociais educativas para pais de crianças com problemas de comportamento. Psicologia: Reflexão e Crítica, 19(3), 407-414. doi:10.1590/S010279722006000300009

Renk, K., \& Phares, V. (2004). Cross-informant ratings of social competence in children and adolescents. Clinical Psychology Review, 24, 239-254. doi:10.1016/j.cpr.2004.01.004

Silva, M. C. R., Montiel, J. M., Pires, S. D., \& Bartholomeu, D. (2010). Teste de desempenho em leitura, escrita e matemática para $o$ ensino Fundamental. Manuscrito não publicado, Laboratório de Psicodiagnóstico e Neurociências Cognitivas, Unisal - Centro Universitário Salesiano de São Paulo, Americana, SP, Brasil.

Stone, W. L., \& La Greca, A. M. (1990). The social status of children with LD: A reexamination. Journal of Learning Disabilities, 23, 32-37. doi:10.1177/002221949002300109

Swanson, H. L., \& Malone, S. (1992). Social skills and learning disabilities: A meta-analysis of the literature. School Psychology Review, 21(3), 427-443.

Tasca, M. (2002). Interferência da língua falada na escrita das séries iniciais: O papel de fatores linguísticos e sociais. Porto Alegre, RS: Editora Universitária da Pontifícia Universidade Católica do Rio Grande do Sul. 
Vaughn, S., \& Hogan, A. (1990). Social competence and learning disabilities: A prospective study. In H. L. Swanson \& B. K. Keogh (Eds.), Learning disabilities: Theoretical and research issues (pp. 175-191). Hillsdale, NJ: Erlbaum.

Vaughn, S., McIntosh, R., \& Spencer-Rowe, J. (1991). Peer rejection is a stubborn thing: Increasing peer acceptance of rejected students with learning disabilities. Learning Disabilities Research \& Practice, 6, 83-88.

Witter, G. P. (1996). Fatores socioculturais e leitura: Estudo da produção arrolada no ASIRR (1989/1994). Estudos de Psicologia (Campinas), 13, 49-56.
Yavas, F., \& Haase, V. G. (1988). Consciência fonêmica em crianças na fase de alfabetização. Letras de Hoje, 23(4), 31-55.

Recebido: 12/11/2014

$1^{a}$ revisão: $10 / 07 / 2015$

$2^{a}$ revisão: $1 \% 09 / 2015$

Aceite final: 19/11/2015 